\section{Ratio of the Magnetic Moments of Proton and Diplon}

RECENT investigations of Farkas and Sachsse ${ }^{1}$ have shown that the ortho $\mathbf{H}_{2} \leftrightarrows$ para $\mathbf{H}_{2}$ transformation is catalysed by paramagnetic gases such as oxygen or nitric oxide. This effect is due to the inhomogeneous magnetic field of the oxygen or nitric oxide molecules acting on the magnetic moment of the protons. The fact that a similar process is observed in the case of diplogen shows that the magnetic moment is also in this case different from zero. From the ratio of the reaction velocities in hydrogen and diplogen, A. Farkas, L. Farkas and P. Harteck ${ }^{2}$ have calculated, using the theory of Wigner $^{3}$, the ratio of the nuclear magnetic moments. They found the magnetic moment of a diplon to be $\mathbf{5 . 5}$ times smaller than that of a proton.

In an investigation which will be published shortly, we have considered some details of the theory of this reaction in order to determine with the greatest possible accuracy the ratio of the magnetic moments. Owing to the special interest in the numerical value of the magnetic moment of the diplon, we mention here the main results.

In the paper by A. Farkas, L. Farkas and P. Harteck, the influence of the different mechanical nuclear moments on the transition probabilities was not taken into account. Including this effect in the calculations, one can show that the probabilities of ortho $\rightarrow$ para and para $\rightarrow$ ortho transitions are propor-

tional to $\left(\frac{S}{2 S+1}\right)\left(\frac{2 S+1}{S}\right)^{2}$ and $\left(\frac{S+1}{2 S+1}\right)\left(\frac{2 S+1}{S}\right)^{2}$

respectively, where $S$ is the nuclear spin. Using the values $S=\frac{1}{2}$ for a proton and $S=1$ for a diplon, we obtain for the ratio of the magnetic moments the value 4 instead of $\mathbf{5} \cdot \mathbf{5}$.

In Wigner's calculations, however, rather special assumptions concerning the spatial distribution of the perturbing forces are involved, so that this new value is also to be regarded as tentative. Therefore, we should like to direct attention to the fact that results of greater reliability can be obtained if the experiments are performed at the absolute temperature $T$ for $\mathrm{H}_{2}$ and at $T / 2$ for $\mathrm{D}_{2}$. In this case nuclear spins $S$, magnetic moments $\mu$ and reaction velocities $k$, which are to be compared at the same molar volumes, are connected by the simple relation :

$$
\left[\frac{2 \mu_{D} S_{P}\left(2 S_{D}+1\right)}{\mu_{P} S_{D}\left(2 S_{P}+1\right)}\right]^{2}=2 \frac{k^{T / 2} D}{k^{T}{ }_{P}}
$$

This formula can be derived without assuming any special mechanism of collision, and by neglecting only the minor effects of the intermolecular Van der Waals forces and of the motion of the paramagnetic molecules. Therefore the ratio $\mu_{D} / \mu_{P}$ can be obtained with an accuracy of a few per cent as soon as the reaction velocities $k^{T} P$ and $k^{T / 2_{D}}$ have been measured.

\section{F. KALOKAR. \\ E. TelLer.}

Institute for Theoretical Physics, Copenhagen. June 30.

${ }^{1}$ Sitz. Ber. Preuss. Akad. Wiss. Berlin, 268; 1933. Z. phys. Chem., B, 23, 1, $19 ; 1933$

2 Proc. Roy. Soc., A, 144, 481 ; 1934.

${ }^{3}$ Z. phys. Chem., B, 23, 28; 1933.

\section{X-Irradiation of Fused Silica}

IN experiments which involved the exposure of fused silica to X-rays, it was observed that the fused silica discoloured. The colour was a very dark violet, and disappeared on heating. The silica was of the clearest and most homogeneous optical quality and presumably very pure. We thought it conceivable, therefore, that the coloration might be due to a reduction of silica to silicon and, since silicon is an excellent reflector in the ultra-violet, that the discoloured irradiated surface might reflect better in the ultra-violet than an unirradiated surface.

Part of the surface of a piece of fused silica was, therefore, irradiated for four hours by radiation from a Shearer tube with a silver target carrying five milliamperes at 70 kilovolts. The relative reflection from the irradiated and clear parts was found by illuminating with light from a quartz mercury vapour lamp, and measuring the intensity of the reflected beam by a photocell with a sodium cathode and a quartz window combined with a Wratten filter No. $18 \mathrm{a}$ which transmits only over the range $3000-4000$ A. The intensity of the beam reflected from the irradiated part was nearly six per cent greater than that from the clear part. Irradiation for a further four hours was carried out, and the irradiated part was then found to reflect above nine per cent more than the clear part. To test whether similar properties appear in the visible, a filament lamp was used as a source and the reflected light was measured with a photronic cell the maximum sensitivity of which was in the region of $0 \cdot 6 \mu$. It was found that the reflection from the irradiated part was not measurably greater than that from the clear part.

A piece of crystalline quartz was similarly irradiated for a total of eight hours. Only a very slight discoloration occurred in this case, and experiments showed that there was no detectable difference between the reflecting powers of the irradiated and non-irradiated parts of the surface.

It is intended to carry out the measurements on reflection farther into the ultra-violet, including the vacuum region.

\section{F. TwYMan \\ F. Brech.}

Research Department,

Adam Hilger, Limited, 98, Kings Road, Camden Road, London, N.W.I. July 11.

\section{Glutathione and Vitamin C in the Crystalline Lens}

Estimations of the ascorbic acid (vitamin C) content of the crystalline lens, by the indophenol titration method of Tilman, indicate that the crystalline lens contains about $0 \cdot 26-0 \cdot 46$ milligrams ascorbic acid per gram of lens. Von Euler and Martius ${ }^{1}$, Birch and Dann ${ }^{2}$, and Müller $^{3}$ are of opinion that a considerable amount of the iodine-reducing substances in the crystalline lens, hitherto considered to be glutathione, is actually ascorbic acid.

Because of the importance of these facts in the consideration of the etiology of cataract, I should like to point out three facts obtained by our study of the sulphhydryl system of the crystalline lens- 